FACTA UNIVERSITATIS

Series: Mechanical Engineering Vol. 18, No 4, 2020, pp. 595 - 610

https://doi.org/10.22190/FUME200416041B

Original scientific paper

\title{
MATHEMATICAL MODELING OF THE INFLUENCE PARAMETERS DURING FORMATION AND PROPAGATION OF THE LÜDERS BANDS
}

\author{
Tin Brlić ${ }^{1}$, Stoja Rešković ${ }^{1}$, Zoran Jurković², Gordan Janeš ${ }^{3}$ \\ ${ }^{1}$ University of Zagreb, Faculty of Metallurgy, Croatia \\ ${ }^{2}$ University of Rijeka, Faculty of Engineering, Croatia \\ ${ }^{3}$ University of Rijeka, Center for Advanced Computing and Modeling, Croatia
}

\begin{abstract}
In this study, an analysis of the influence parameters measured by the static tensile test, thermography and digital image correlation was performed during formation and propagation of the Lüders bands. A new approach to the prediction of stresses, maximum temperature changes and strains during the Lüders band formation and propagation is proposed in this paper. Application of the obtained mathematical models of influence parameters gives a clear insight into the behavior of niobium microalloyed steel at the beginning of the plastic flow, which can improve product quality and reduce costs during the forming of microalloyed steels with the appearance of the Lüders bands. The obtained models of influential parameters during formation and propagation of the Lïders bands have been developed by the regression analysis method. The proposed mathematical models showed low deviations of calculated results ranging from $1.34 \%$ to $12.37 \%$. The local stress amounts, important in the forming of microalloyed steels since indicating surface roughness and plastic flow possibilities during the Lüders band propagation, are obtained by the mathematical model. It was found that stress amounts increase during the Lüders band propagation in the area behind the Lüders band front. The difference in stress amount between the start of the Lüders band propagation and advanced Lüders band propagation is $25.53 \mathrm{MPa}$.
\end{abstract}

Key Words: Lüders Bands, Regression Analysis, Microalloyed Steel, Thermography, Digital Image Correlation

Received April 16, 2020 / Accepted September 02, 2020

Corresponding author: Tin Brlić

University of Zagreb Faculty of Metallurgy, Aleja narodnih heroja 3, 44000 Sisak, Croatia

E-mail: tbrlic@simet.unizg.hr

ㄷ 2020 by University of Niš, Serbia | Creative Commons Licence: CC BY-NC-ND 


\section{INTRODUCTION}

Mathematical modeling [1] and optimization of process parameters [2] are often used for improving the quality of products as well as reducing costs of the production process for various metallic materials. The appearance of the Lüders bands presents a problem for the product quality during the forming process [3]. Consequently, mathematical modeling was used in order to predict behavior of the Lüders bands during cold deformation of metallic materials [4]. It is well known that niobium microalloyed steels show inhomogeneous deformations, i.e. Lüders bands, at the start of plastic flow during cold deformation [5], which can be a significant problem during the deformation process of microalloyed steels.

It is possible to improve product quality and reduce production process costs by developing mathematical models of influential parameters. Given that, it is not necessary to carry out additional experimental tests that require additional costs since the values of significant parameters obtained by the mathematical models can be predicted. The formation and propagation of the Lüders bands in metallic materials showed a difference in the values of influential parameters of stress, temperature changes [6] and strains behind the Lüders band front [7]. Since changes of influence parameters can be more or less pronounced, it is important to analyze them in detail and describe with mathematical models in order to give a clear insight of value changes of the parameters during the formation and propagation of the Lüders bands.

Static tensile test, thermography and digital image correlation are confirmed as reliable methods for determining the stress, temperature changes and strain amounts [8] during the formation and propagation of the Lüders bands. It is well known that stress values can be measured from the force-elongation diagram by static tensile test. Values of temperature changes and their distribution in Lüders bands can be determined with high accuracy using thermography. Strain values and their distribution can be determined by digital image correlation during static tensile testing. Therefore, it can be concluded that using thermography and digital image correlation can determine temperature changes and strain values which can be compared with the stress values obtained by the static tensile test.

Previous research has shown that mathematical modeling is often used to describe processes during deformation under various metal forming technologies [9].

Bhirud and Gawande [10] used the regression analysis method to determine the mathematical model for temperature increase of work piece during final milling. Results of the obtained mathematical model are compared with the measured results of temperature increase of the work piece and a confirmation test is conducted for the validation of the predicted results of temperature increase. The regression analysis method proved to be suitable for a better insight into the tensile behavior of AISI 316 stainless steel during which temperature change and strain rate have been associated by corresponding empirical equations [11]. Nasri et al. [12] have shown usability of the multiple regression method during sheet metal forming formability for comparison between experimental results and model predictions.

Jurković et al. [13] conducted the tests of rolling steel sheets where the output parameter, forming force-roll load, are mathematically modeled by the regression analysis of various input parameters such as tensile strength, sheet thickness and sheet width where regression analysis proved to be a reliable and accurate method. The research [14] confirms the regression analysis method as a very good method for obtaining mathematical models in determining the influence of cutting speed, feed rate and depth of cut on cutting tool vibration in different directions on carbon steel. The obtained regression mathematical models for vibration of cutting tool with machining parameters have shown a very good match between the results predicted from the developed models and the experimental ones. Other studies [15] 
confirmed regression analysis as a reliable method for obtaining appropriate mathematical models of selected output parameters. The significance of the process parameter influence of spindle rotation speed, feed speed and rolling-beating mode during cold rolling-beating forming process on ASTM 1045 steel for obtaining mathematical models of forming forces and forming qualities objectives by regression analysis is determined in [16].

Mathematical multiple linear regression analysis of mechanical properties of potential weld joints, such as yield strength, ultimate tensile strength and percentage elongation, on aluminum alloy AA 6061 during manufacturing conditions is conducted in [17]. Models have proven to be very accurate in prediction of mechanical properties which can greatly assist engineers in proper planning and simultaneously improve efficiency of aluminum alloy welding operations.

The phenomenon of the Lüders bands tends to be mathematically described for different steels [18]. Studies [4, 19] used developed models by regression analysis for different influencing parameters in low carbon steel with Lüders bands. Geometrical properties such as length, width and depth of the Lüders bands were examined which appeared on the tinplates during the cold stamping process. The predicted model results of influencing parameters showed good agreement with the measured results obtained by experimental tests. The tendency of Sadowski et al. [20] to model the phenomenon of Lüders bands by regression analysis is manifested where they sought to model different yield plateau gradients for different structural carbon steels. Rešković et al. [21] used factorial experiment with repeated measurements to determine influence of different factors at the proportionality limit after which Lüders bands appear in niobium microalloyed steel during cold deformation. They concluded that the deformation degree has the greatest influence on the proportionality limit during cold drawing of niobium microalloyed steel tubes.

In the local area of maximum temperature changes and maximum strains behind the Lüders front detailed influence of individual influencing parameters is not mathematically described. Therefore, the aim of this paper is to develop mathematical models for influential parameters $\Delta T_{\max }$ (maximum temperature change), $\sigma$ (stress) and $\varepsilon_{\max }$ (maximum strain) to predict the behavior of niobium microalloyed steel in the area behind the Lüders front which shows the greatest temperature and strain changes during cold deformation.

\section{EXPERIMENTAL WORK}

Measured experimental results of influencing parameters during the formation and propagation of the Lüders bands were obtained on microalloyed steel with $0.048 \% \mathrm{Nb}$. The chemical composition of niobium microalloyed steel is shown in Table 1.

Influential parameter of the stress was obtained by static tensile testing at the stretching rates of $5 \mathrm{~mm} / \mathrm{min}, 20 \mathrm{~mm} / \mathrm{min}$ and $50 \mathrm{~mm} / \mathrm{min}$ corresponding to strain rates of $0.0018 \mathrm{~s}^{-1}$, $0.007 \mathrm{~s}^{-1}$ and $0.0185 \mathrm{~s}^{-1}$. Static tensile tests were carried out on the tensile machine WPM EU $40 \mathrm{mod}$ with a nominal force value $400 \mathrm{kN}$. Determination of the local maximum temperature changes and maximum strain values was carried out by infrared camera JENOPTIK VarioCAM®M82910 and digital camera Blackfly S Color (FLIR).

Values of influential parameters were measured using the aforementioned research methods during formation and propagation of the Lüders bands. The parameters of maximum localized temperature change $\left(\Delta T_{\max }\right)$, maximum strains $\left(\varepsilon_{\max }\right)$ and stress values $(\sigma)$ were measured using thermography, digital image correlation and the static tensile test. 
In addition to the influential parameters, different strain rates during static tensile testing were also considered.

The measurements of parameters $\Delta T_{\max }$ and $\varepsilon_{\max }$ were performed in the area behind the Lüders band front during formation and propagation of the Lüders bands since the largest changes of the analyzed parameters were measured in this area. Other parameters during the experimental tests that may have influenced the accuracy of the mathematical model were kept constant during the static tensile test. The levels of the minimum and maximum values of the input parameters were determined during formation and propagation of the Lüders bands when determining $\sigma$ as output parameter, Table 2, and $\Delta T_{\max }, \varepsilon_{\max }$ as output parameters in Tables 3 and 4.

\subsection{Regression analysis method}

This paper presents modeling of output parameters $\sigma, \varepsilon_{\max }$ and $\Delta T_{\max }$ using a mathematical statistical method of regression analysis. It is possible to define the relationship between the output effects of process $(y)$ and input variables $\left(x_{i}\right)$ by regression analysis. Mathematical modeling of influential parameters during the formation and propagation of the Lüders bands by the regression analysis is performed by the second order polynomial model using Eq. (1):

$$
\begin{gathered}
y=b_{0} x_{0}+b_{1} x_{1}+b_{2} x_{2}+b_{3} x_{3}+b_{11} x_{1}{ }^{2}+b_{22} x_{2}{ }^{2}+b_{33} x_{3}{ }^{2}+ \\
b_{12} x_{1} x_{2}+b_{13} x_{1} x_{3}+b_{23} x_{2} x_{3}+b_{123} x_{1} x_{2} x_{3}
\end{gathered}
$$

where $x_{i}$ are the variable parameters of process $\mathrm{i}=0,1,2, \ldots, \mathrm{k}$ and $b_{i}$ are the model coefficients.

Table 1 Chemical composition of niobium microalloyed steel (wt\%)

\begin{tabular}{ccccccccc}
\hline Element & $\mathrm{C}$ & $\mathrm{Mn}$ & $\mathrm{Si}$ & $\mathrm{P}$ & $\mathrm{S}$ & $\mathrm{Al}$ & $\mathrm{Nb}$ & $\mathrm{N}$ \\
\hline Nb steel & 0.12 & 0.78 & 0.18 & 0.011 & 0.018 & 0.020 & 0.048 & 0.0080 \\
\hline
\end{tabular}

Table 2 Physical values of input parameters for determining output parameter $\sigma$ during the formation and propagation of the Lüders bands at $v=0.007 \mathrm{~s}^{-1}$

\begin{tabular}{lcrrr}
\hline & Parameters & Minimum value & Mean value & Maximum value \\
\hline Formation & $\Delta T_{\max }\left({ }^{\circ} \mathrm{C}\right)$ & -0.47 & 1.26 & 2.05 \\
of the Lüders band & $\varepsilon_{\max }(\mathrm{mm} / \mathrm{mm})$ & 0.0072 & 0.0204 & 0.0336 \\
\hline Propagation & $\Delta T_{\max }\left({ }^{\circ} \mathrm{C}\right)$ & 1.37 & 2.64 & 3.91 \\
of the Lüders band & $\varepsilon_{\max }(\mathrm{mm} / \mathrm{mm})$ & 0.0300 & 0.0396 & 0.0492 \\
\hline
\end{tabular}

Table 3 Physical values of input parameters for determining output parameter $\varepsilon_{\max }$ during the formation and propagation of the Lüders bands

\begin{tabular}{lcrrr}
\hline & Parameters & Minimum value & Mean value & Maximum value \\
\hline \multirow{2}{*}{ Formation of the } & $\Delta T_{\max }\left({ }^{\circ} \mathrm{C}\right)$ & -0.59 & 0.98 & 2.55 \\
Lüders band & $\sigma(\mathrm{MPa})$ & 484.70 & 524.60 & 564.50 \\
& $v\left(\mathrm{~s}^{-1}\right)$ & 0.00180 & 0.01015 & 0.01850 \\
\hline \multirow{2}{*}{ Propagation of the } & $\Delta T_{\max }\left({ }^{\circ} \mathrm{C}\right)$ & 0.350 & 2.285 & 4.220 \\
Lüders band & $\sigma(\mathrm{MPa})$ & 498.30 & 529.95 & 561.60 \\
& $v\left(\mathrm{~s}^{-1}\right)$ & 0.00180 & 0.01015 & 0.01850 \\
\hline
\end{tabular}


Table 4 Physical values of input parameters for determining output parameter $\Delta T_{\max }$ during the Lüders bands propagation

\begin{tabular}{lccc}
\hline Parameters & Minimum value & Mean value & Maximum value \\
\hline$\varepsilon_{\max }(\mathrm{mm} / \mathrm{mm})$ & 0.02790 & 0.03855 & 0.04920 \\
$\sigma(\mathrm{MPa})$ & 498.30 & 529.95 & 561.60 \\
$v\left(\mathrm{~s}^{-1}\right)$ & 0.00180 & 0.01015 & 0.01850 \\
\hline
\end{tabular}

Multiregression coefficient, $R$, determined by Eq. (2) is very important for the quality of the mathematical models obtained by the regression analysis method:

$$
\mathrm{R}=\sqrt{1-\frac{\sum_{\mathrm{j}=1}^{\mathrm{N}}\left(Y_{j}^{E}-Y_{j}^{R}\right)^{2}}{\sum_{\mathrm{j}=1}^{\mathrm{N}}\left(Y_{j}^{E}-\bar{Y}_{j}^{E}\right)^{2}}}
$$

where $Y_{j}^{E}$ are the experimental values, $Y_{j}^{R}$ the calculated values and $\bar{Y}^{E}=\frac{\sum_{j=1}^{N} Y_{j}^{E}}{N}$ the arithmetic mean of all experimental results.

Determination coefficient, $R^{2}$, is determined by calculated multiregression coefficient $R$. The general form of a second order polynomial model with interacting factors will take the following forms with respect to the parameters analyzed in this paper.

Model for output parameter $\sigma$ :

- for the formation and propagation of the Lüders band at $v=0.007 \mathrm{~s}^{-1}$ :

$$
y(\sigma)=b_{0}+b_{1} \Delta T_{\max }+b_{2} \varepsilon_{\max }+b_{11} \Delta T_{\max }^{2}+b_{22} \varepsilon_{\max }^{2}+b_{12} \Delta T_{\max } \varepsilon_{\max }
$$

The general form of a mathematical model for output parameters $\Delta T_{\max }$ and $\varepsilon_{\max }$ denoted by $z$ :

- for the formation and propagation of the Lüders band:

$$
y(z)=b_{0}+b_{1} z+b_{2} \sigma+b_{3} v+b_{11} z_{\text {max }}^{2}+b_{22} \sigma^{2}+b_{33} v^{2}+b_{12} z \sigma+b_{13} z v+b_{23} \sigma v+b_{123} z \sigma v
$$

\section{RESULTS AND DISCUSSION}

Mathematical modeling of influence parameters $\left(\varepsilon_{\max }, \Delta T_{\max }, \sigma\right)$ using regression analysis was performed during the formation and propagation of the Lüders bands at the start of the plastic flow in niobium microalloyed steel during cold deformation. The experimental results of the maximum local temperature changes and strains in the area behind the Lüders band front during formation and propagation of the Lüders bands were performed in the area according to Fig. 1. Experimental results obtained by using static tensile testing, thermography and digital image correlation are shown in Table 5 for output parameter $\sigma$, and in Tables 6-8 for output parameters $\left(\varepsilon_{\max }, \Delta T_{\max }\right)$. 


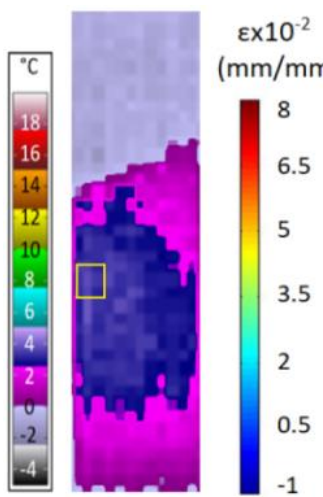

a)

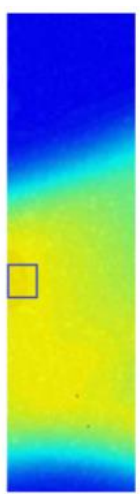

b)

Fig. 1 The area of measurements of: a) maximum temperature change and b) maximum strain in the area behind the Lüders front

Table 5 Experimental results of input parameters $\left(\Delta T_{\max }, \varepsilon_{\max }\right)$ and output parameter $\sigma$ obtained by measured values during formation and propagation of the Lüders bands at $v=0.007 \mathrm{~s}^{-1}$

\begin{tabular}{|c|c|c|c|c|c|c|}
\hline \multirow{3}{*}{ 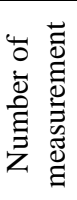 } & \multicolumn{3}{|c|}{ Formation of the Lüders band } & \multicolumn{3}{|c|}{ Propagation of the Lüders band } \\
\hline & \multicolumn{2}{|c|}{ Input parameters } & \multirow{2}{*}{$\begin{array}{c}\begin{array}{c}\text { Output } \\
\text { parameter }\end{array} \\
\begin{array}{c}\sigma \\
(\mathrm{MPa})\end{array}\end{array}$} & \multicolumn{2}{|c|}{ Input parameters } & \multirow{2}{*}{$\begin{array}{c}\begin{array}{c}\text { Output } \\
\text { parameter }\end{array} \\
\sigma \\
\begin{array}{c}\sigma \\
(\mathrm{MPa})\end{array}\end{array}$} \\
\hline & $\begin{array}{c}\Delta T_{\max } \\
\left({ }^{\circ} \mathrm{C}\right)\end{array}$ & $\begin{array}{c}\varepsilon_{\max } \\
(\mathrm{mm} / \mathrm{mm})\end{array}$ & & $\begin{array}{c}\Delta T_{\max } \\
\left({ }^{\circ} \mathrm{C}\right)\end{array}$ & $\begin{array}{c}\varepsilon_{\max } \\
(\mathrm{mm} / \mathrm{mm})\end{array}$ & \\
\hline 1 & -0.47 & 0.0076 & 543.3 & 1.37 & 0.0336 & 498.3 \\
\hline 2 & -0.35 & 0.0072 & 530.6 & 1.72 & 0.0374 & 498.3 \\
\hline 3 & 0.17 & 0.0137 & 497.5 & 1.97 & 0.0378 & 500.6 \\
\hline 4 & 0.17 & 0.0094 & 535.8 & 2.01 & 0.0316 & 531.1 \\
\hline 5 & 0.42 & 0.0113 & 552.0 & 2.05 & 0.0300 & 522.6 \\
\hline 6 & 0.67 & 0.0211 & 506.9 & 2.16 & 0.0412 & 512.3 \\
\hline 7 & 1.07 & 0.0267 & 499.0 & 2.19 & 0.0388 & 510.0 \\
\hline 8 & 1.23 & 0.0205 & 523.2 & 2.22 & 0.0398 & 510.8 \\
\hline 9 & 1.37 & 0.0336 & 498.3 & 2.46 & 0.0386 & 540.7 \\
\hline 10 & 1.67 & 0.0234 & 536.0 & 2.71 & 0.0373 & 521.3 \\
\hline 11 & 2.01 & 0.0316 & 531.1 & 2.73 & 0.0416 & 545.9 \\
\hline 12 & 2.05 & 0.0300 & 522.6 & 2.76 & 0.0434 & 549.3 \\
\hline 13 & & & & 2.80 & 0.0441 & 554.4 \\
\hline 14 & & & & 3.04 & 0.0427 & 528.5 \\
\hline 15 & & & & 3.30 & 0.0479 & 558.7 \\
\hline 16 & & & & 3.42 & 0.0439 & 524.5 \\
\hline 17 & & & & 3.65 & 0.0481 & 526.7 \\
\hline 18 & & & & 3.91 & 0.0492 & 521.2 \\
\hline
\end{tabular}


Table 6 Experimental results of input parameters $\left(\Delta T_{\max }, \sigma, v\right)$ and output parameter $\varepsilon_{\max }$ obtained by measured values during formation of the Lüders bands

\begin{tabular}{|c|c|c|c|c|c|c|c|c|c|}
\hline \multirow{2}{*}{ 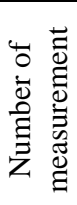 } & \multicolumn{3}{|c|}{ Input parameters } & \multirow{2}{*}{$\begin{array}{c}\begin{array}{c}\text { Output } \\
\text { parameter }\end{array} \\
\varepsilon_{\max } \\
(\mathrm{mm} / \mathrm{mm})\end{array}$} & \multirow{2}{*}{ 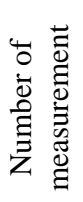 } & \multicolumn{3}{|c|}{ Input parameters } & \multirow{2}{*}{$\begin{array}{c}\begin{array}{c}\text { Output } \\
\text { parameter }\end{array} \\
\varepsilon_{\max } \\
(\mathrm{mm} / \mathrm{mm})\end{array}$} \\
\hline & $\begin{array}{c}\Delta T_{\max } \\
\left({ }^{\circ} \mathrm{C}\right)\end{array}$ & $\begin{array}{c}\sigma \\
(\mathrm{MPa})\end{array}$ & $\begin{array}{c}v \\
\left(\mathrm{~s}^{-1}\right)\end{array}$ & & & $\begin{array}{c}\Delta T_{\max } \\
\left({ }^{\circ} \mathrm{C}\right)\end{array}$ & $\begin{array}{c}\sigma \\
(\mathrm{MPa})\end{array}$ & $\begin{array}{c}v \\
\left(\mathrm{~s}^{-1}\right)\end{array}$ & \\
\hline 1 & -0.59 & 489.4 & 0.0018 & 0.0057 & 19 & 0.67 & 506.9 & 0.0070 & 0.0211 \\
\hline 2 & -0.55 & 484.7 & 0.0018 & 0.0073 & 20 & 0.74 & 555.9 & 0.0185 & 0.0199 \\
\hline 3 & -0.47 & 493.3 & 0.0018 & 0.0060 & 21 & 0.80 & 512.6 & 0.0018 & 0.0308 \\
\hline 4 & -0.47 & 543.3 & 0.0070 & 0.0076 & 22 & 0.81 & 539.8 & 0.0018 & 0.0350 \\
\hline 5 & -0.44 & 508.3 & 0.0018 & 0.0069 & 23 & 0.99 & 545.3 & 0.0185 & 0.0227 \\
\hline 6 & -0.35 & 530.6 & 0.0070 & 0.0072 & 24 & 1.07 & 499.0 & 0.0070 & 0.0267 \\
\hline 7 & -0.31 & 545.6 & 0.0185 & 0.0059 & 25 & 1.23 & 523.2 & 0.0070 & 0.0205 \\
\hline 8 & -0.29 & 515.9 & 0.00 & 0.0127 & 26 & 1.37 & 498.3 & & 0.0336 \\
\hline 9 & -0.28 & 516.9 & 0.0185 & 0.0080 & 27 & 1.51 & 539.5 & 0.0 & 0.0325 \\
\hline 10 & -0.24 & 509.4 & 0.0185 & 0.0086 & 28 & 1.66 & 549.1 & 85 & 0.0324 \\
\hline 11 & 0.02 & 510.9 & 0.0018 & 0.0172 & 29 & 1.67 & 536.0 & 0.0070 & 0.0234 \\
\hline 12 & 0.17 & 497.5 & 0.0070 & 0.0137 & 30 & 1.69 & 564.5 & 0.0185 & 0.0324 \\
\hline 13 & 0.17 & 535.8 & 0.0070 & 0.0094 & 31 & 2.01 & 531.1 & 0.0070 & 0.0316 \\
\hline 14 & 0.34 & 536.7 & 0.0018 & 0.0198 & 32 & 2.05 & 522.6 & 0.0070 & 0.0300 \\
\hline 15 & 0.39 & 509.1 & 0.0018 & 0.0279 & 33 & 2.06 & 535.0 & 0.0185 & 0.0325 \\
\hline 16 & 0.42 & 552.0 & 0.0070 & 0.0113 & 34 & 2.16 & 536.4 & 0.0185 & 0.0380 \\
\hline 17 & 0.5 & 512.1 & 0.0018 & 0.0215 & 35 & 2.53 & 528.1 & 0.0185 & 0.0400 \\
\hline 18 & 0.62 & 536.1 & 0.0018 & 0.0280 & 36 & 2.55 & 540.3 & 0.0185 & 0.0412 \\
\hline
\end{tabular}

Mathematical models for the influence parameters were obtained by using measured experimental values of $\Delta T_{\max }, \varepsilon_{\max }$ and $\sigma$ during formation and propagation of the Lüders bands. The experimental measured values were used to develop the equations of the second order polynomial model. The results of the data processing by regression analysis performed in Microsoft $\AA$ Excel are shown in Tables 9-12.

Regression analysis of influence parameters $\left(\varepsilon_{\max }, \Delta T_{\max }, \sigma\right)$ based on measured values during the formation and propagation of the Lüders bands revealed high values of multiregression coefficient $(R)$ and determination coefficient $\left(R^{2}\right)$ which confirms that the obtained mathematical models can be considered reliable. The results of the multiregression coefficients and determination coefficients obtained by regression analysis of the influencing parameters are shown in Table 9.

High values of the multiregression coefficients and determination coefficients from the regression analysis were obtained. Mathematical models for the influential parameters during formation and propagation of the Lüders bands are shown by the following equations:

- mathematical model of the influential parameter $\sigma$ during formation of the Lüders bands at $v=0.007 \mathrm{~s}^{-1}$ :

$\sigma=622.9334+75.945 \Delta T_{\max }-12078.3 \varepsilon_{\max }+26.281 \Delta T_{\max }^{2}+281771 \varepsilon_{\max }^{2}-4063.16 \Delta T_{\max } \varepsilon_{\max }$

- mathematical model of influential parameter $\sigma$ during propagation of the Lüders bands at $v=0.007 \mathrm{~s}^{-1}$ :

$\sigma=640.894-90.392 \Delta T_{\max }-1049.07 \varepsilon_{\max }-86.8013 \Delta T_{\max }^{2}-399310 \varepsilon_{\max }^{2}+13425.7 \Delta T_{\max } \varepsilon_{\max }$ 
- mathematical model of influential parameter $\varepsilon_{\max }$ during formation of the Lüders bands:

$\varepsilon_{\max }=1.0398+0.178 \Delta T_{\max }-0.004 \sigma+4.866 v-0.00078 \Delta T_{\max }^{2}+0.00000401 \sigma^{2}+144.558 v^{2}-$

$$
0.00031 \Delta T_{\max } \sigma-8.807 \Delta T_{\max } v-0.01577 \sigma v+0.0169 \Delta T_{\max } \sigma v
$$

Table 7 Experimental results of input parameters $\left(\Delta T_{\max }, \sigma, v\right)$ and output parameter $\varepsilon_{\max }$ obtained by measured values during propagation of the Lüders bands

\begin{tabular}{|c|c|c|c|c|c|c|c|c|c|}
\hline \multirow{2}{*}{ 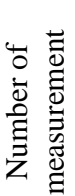 } & \multicolumn{3}{|c|}{ Input parameters } & $\begin{array}{c}\text { Output } \\
\text { parameter }\end{array}$ & \multirow{2}{*}{ 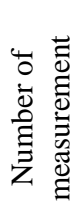 } & \multicolumn{3}{|c|}{ Input parameters } & $\begin{array}{c}\text { Output } \\
\text { parameter }\end{array}$ \\
\hline & $\begin{array}{c}\Delta T_{\max } \\
\left({ }^{\circ} \mathrm{C}\right)\end{array}$ & $\sigma(\mathrm{MPa})$ & $\begin{array}{c}v \\
\left(\mathrm{~s}^{-1}\right)\end{array}$ & $\begin{array}{c}\varepsilon_{\max } \\
(\mathrm{mm} / \mathrm{mm})\end{array}$ & & $\begin{array}{c}\Delta T_{\max }\left({ }^{\circ}\right. \\
\mathrm{C})\end{array}$ & $\sigma(\mathrm{MPa})$ & $\begin{array}{c}v \\
\left(\mathrm{~s}^{-1}\right)\end{array}$ & $\begin{array}{c}\varepsilon_{\max } \\
(\mathrm{mm} / \mathrm{mm})\end{array}$ \\
\hline
\end{tabular}

\begin{tabular}{|c|c|c|c|c|c|c|c|c|c|}
\hline 1 & 0.35 & 508.6 & 0.0018 & 0.0309 & 28 & 2.27 & 510.8 & 0.0018 & 0.0405 \\
\hline 2 & 0.39 & 509.1 & 0.0018 & 0.0279 & 29 & 2.46 & 540.7 & 0.0070 & 0.0386 \\
\hline 3 & 0.61 & 509.8 & 0.0018 & 0.0325 & 30 & 2.55 & 535.6 & 0.0185 & 0.0392 \\
\hline 4 & 0.75 & 511.4 & 0.0018 & 0.0331 & 31 & 2.71 & 521.3 & 0.0070 & 0.0373 \\
\hline 5 & 0.80 & 512.6 & 0.0018 & 0.0308 & 32 & 2.73 & 545.9 & 0.0070 & 0.0416 \\
\hline 6 & 0.81 & 539.8 & 0.0018 & 0.0350 & 33 & 2.76 & 549.3 & 0.0070 & 0.0434 \\
\hline 7 & 0.98 & 543.3 & 0.0018 & 0.0379 & 34 & 2.80 & 554.4 & 0.0070 & 0.0441 \\
\hline 8 & 1.00 & 509.7 & 0.0018 & 0.0336 & 35 & 2.86 & 529.0 & 0.0185 & 0.0378 \\
\hline 9 & 1.11 & 510.1 & 0.0018 & 0.0344 & 36 & 3.03 & 526.7 & 0.0185 & 0.0414 \\
\hline 10 & 1.18 & 549.0 & 0.0018 & 0.0432 & 37 & 3.04 & 528.5 & 0.0070 & 0.0427 \\
\hline 11 & 1.19 & 547.3 & 0.0018 & 0.0409 & 38 & 3.08 & 539.2 & 0.0185 & 0.0408 \\
\hline 12 & 1.37 & 498.3 & 0.0070 & 0.0336 & 39 & 3.30 & 558.7 & 0.0070 & 0.0479 \\
\hline 13 & 1.46 & 545.3 & 0.0018 & 0.0448 & 40 & 3.35 & 540.5 & 0.0185 & 0.0429 \\
\hline 14 & 1.50 & 510.6 & 0.0018 & 0.0363 & 41 & 3.39 & 533.4 & 0.0185 & 0.0424 \\
\hline 15 & 1.55 & 510.5 & 0.0018 & 0.0368 & 42 & 3.42 & 524.5 & 0.0070 & 0.0439 \\
\hline 16 & 1.72 & 498.3 & 0.0070 & 0.0374 & 43 & 3.60 & 527.9 & 0.0185 & 0.0432 \\
\hline 17 & 1.82 & 509.7 & 0.0018 & 0.0377 & 44 & 3.62 & 545.5 & 0.0185 & 0.0449 \\
\hline 18 & 1.91 & 510.8 & 0.0018 & 0.0386 & 45 & 3.65 & 526.7 & 0.0070 & 0.0481 \\
\hline 19 & 1.97 & 500.6 & 0.0070 & 0.0378 & 46 & 3.74 & 531.3 & 0.0185 & 0.0442 \\
\hline 20 & 2.01 & 531.1 & 0.0070 & 0.0316 & 47 & 3.84 & 535.7 & 0.0185 & 0.0429 \\
\hline 21 & 2.05 & 522.6 & 0.0070 & 0.0300 & 48 & 3.86 & 557.2 & 0.0185 & 0.0476 \\
\hline 22 & 2.06 & 534.6 & 0.0185 & 0.0402 & 49 & 3.91 & 521.2 & 0.0070 & 0.0492 \\
\hline 23 & 2.07 & 549.0 & 0.0018 & 0.0455 & 50 & 4.05 & 546.0 & 0.0185 & 0.0463 \\
\hline 24 & 2.16 & 512.3 & 0.0070 & 0.0412 & 51 & 4.08 & 561.6 & 0.0185 & 0.0480 \\
\hline 25 & 2.16 & 528.1 & 0.0185 & 0.0324 & 52 & 4.12 & 537.4 & 0.0185 & 0.0436 \\
\hline 26 & 2.19 & 510.0 & 0.0070 & 0.0388 & 53 & 4.18 & 530.9 & 0.0185 & 0.0473 \\
\hline 27 & 2.22 & 510.8 & 0.0070 & 0.0398 & 54 & 4.22 & 544.7 & 0.0185 & 0.0447 \\
\hline
\end{tabular}

- mathematical model of influential parameter $\varepsilon_{\max }$ during propagation of the Lüders bands:

$$
\begin{gathered}
\varepsilon_{\max }=3.036+0.0732 \Delta T_{\max }-0.01168 \sigma-16.120 v+0.00107 \Delta T_{\max }^{2}+0.000011 \sigma^{2}+ \\
50.721 v^{2}-0.00014 \Delta T_{\max } \sigma+4.2917 \Delta T_{\max } v+0.0287 \sigma v-0.00836 \Delta T_{\max } \sigma v
\end{gathered}
$$

- mathematical model of influential parameter $\Delta T_{\max }$ during propagation of the Lüders bands: 


$$
\begin{gathered}
\Delta T_{\max }=-309.167+48.762 \varepsilon_{\max }+1.1811 \sigma+2310.75 v+1210.47 \varepsilon_{\max }^{2}-0.00113 \sigma^{2}- \\
10906.6 v^{2}-0.03997 \varepsilon_{\max } \sigma-77240.3 \varepsilon_{\max } v-3.848 \sigma v+146.69 \varepsilon_{\max } \sigma v
\end{gathered}
$$

Table 8 Experimental results of input parameters $\left(\varepsilon_{\max }, \sigma, v\right)$ and output parameter $\Delta T_{\max }$

\begin{tabular}{|c|c|c|c|c|c|c|c|c|c|}
\hline \multirow{2}{*}{ 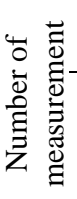 } & \multicolumn{3}{|c|}{ Input parameters } & \multirow{2}{*}{$\begin{array}{c}\begin{array}{c}\text { Output } \\
\text { parameter }\end{array} \\
\begin{array}{c}\Delta T_{\max } \\
\left({ }^{\circ} \mathrm{C}\right)\end{array}\end{array}$} & \multirow{2}{*}{ 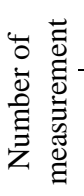 } & \multicolumn{3}{|c|}{ Input parameters } & \multirow{2}{*}{$\begin{array}{c}\begin{array}{c}\text { Output } \\
\text { parameter }\end{array} \\
\begin{array}{c}\Delta T_{\max } \\
\left({ }^{\circ} \mathrm{C}\right)\end{array}\end{array}$} \\
\hline & $\begin{array}{c}\varepsilon_{\max } \\
(\mathrm{mm} / \mathrm{mm})\end{array}$ & $\begin{array}{c}\sigma \\
(\mathrm{MPa})\end{array}$ & $\begin{array}{c}v \\
\left(\mathrm{~s}^{-1}\right)\end{array}$ & & & $\begin{array}{c}\varepsilon_{\max } \\
(\mathrm{mm} / \mathrm{mm})\end{array}$ & $\begin{array}{c}\sigma \\
(\mathrm{MPa})\end{array}$ & $\begin{array}{c}v \\
\left(\mathrm{~s}^{-1}\right)\end{array}$ & \\
\hline 1 & 0.0309 & 508.6 & 0.0018 & 0.35 & 28 & 0.0405 & 510.8 & 0.0018 & 2.27 \\
\hline 2 & 0.0279 & 509.1 & 0.0018 & 0.39 & 29 & 0.0386 & 540.7 & 0.0070 & 2.46 \\
\hline 3 & 0.0325 & 509.8 & 0.0018 & 0.61 & 30 & 0.0392 & 535.6 & 0.0185 & 2.55 \\
\hline 4 & 0.0331 & 511.4 & 0.0018 & 0.75 & 31 & 0.0373 & 521.3 & 0.0070 & 2.71 \\
\hline 5 & 0.0308 & 512.6 & 0.0018 & 0.80 & 32 & 0.0416 & 545.9 & 0.0070 & 2.73 \\
\hline 6 & 0.0350 & 539.8 & 0.0018 & 0.81 & 33 & 0.0434 & 549.3 & 0.0070 & 2.76 \\
\hline 7 & 0.0379 & 543.3 & 0.0018 & 0.98 & 34 & 0.0441 & 554.4 & 0.0070 & 2.80 \\
\hline 8 & 0.0336 & 509.7 & 0.0018 & 1.00 & 35 & 0.0378 & 529.0 & 0.0185 & 2.86 \\
\hline 9 & 0.0344 & 510.1 & 0.0018 & 1.11 & 36 & 0.0414 & 526.7 & 0.0185 & 3.03 \\
\hline 10 & 0.0432 & 549.0 & 0.0018 & 1.18 & 37 & 0.0427 & 528.5 & 0.0070 & 3.04 \\
\hline 11 & 0.0409 & 547.3 & 0.0018 & 1.19 & 38 & 0.0408 & 539.2 & 0.0185 & 3.08 \\
\hline 12 & 0.0336 & 498.3 & 0.0070 & 1.37 & 39 & 0.0479 & 558.7 & 0.0070 & 3.30 \\
\hline 13 & 0.0448 & 545.3 & 0.0018 & 1.46 & 40 & 0.0429 & 540.5 & 0.0185 & 3.35 \\
\hline 14 & 0.0363 & 510.6 & 0.0018 & 1.50 & 41 & 0.0424 & 533.4 & 0.0185 & 3.39 \\
\hline 15 & 0.0368 & 510.5 & 0.0018 & 1.55 & 42 & 0.0439 & 524.5 & 0.0070 & 3.42 \\
\hline 16 & 0.0374 & 498.3 & 0.0070 & 1.72 & 43 & 0.0432 & 527.9 & 0.0185 & 3.60 \\
\hline 17 & 0.0377 & 509.7 & 0.0018 & 1.82 & 44 & 0.0449 & 545.5 & 0.0185 & 3.62 \\
\hline 18 & 0.0386 & 510.8 & 0.0018 & 1.91 & 45 & 0.0481 & 526.7 & 0.0070 & 3.65 \\
\hline 19 & 0.0378 & 500.6 & 0.0070 & 1.97 & 46 & 0.0442 & 531.3 & 0.0185 & 3.74 \\
\hline 20 & 0.0316 & 531.1 & 0.0070 & 2.01 & 47 & 0.0429 & 535.7 & 0.0185 & 3.84 \\
\hline 21 & 0.0300 & 522.6 & 0.0070 & 2.05 & 48 & 0.0476 & 557.2 & 0.0185 & 3.86 \\
\hline 22 & 0.0402 & 534.6 & 0.0185 & 2.06 & 49 & 0.0492 & 521.2 & 0.0070 & 3.91 \\
\hline 23 & 0.0455 & 549.0 & 0.0018 & 2.07 & 50 & 0.0463 & 546.0 & 0.0185 & 4.05 \\
\hline 24 & 0.0412 & 512.3 & 0.0070 & 2.16 & 51 & 0.0480 & 561.6 & 0.0185 & 4.08 \\
\hline 25 & 0.0324 & 528.1 & 0.0185 & 2.16 & 52 & 0.0436 & 537.4 & 0.0185 & 4.12 \\
\hline 26 & 0.0388 & 510.0 & 0.0070 & 2.19 & 53 & 0.0473 & 530.9 & 0.0185 & 4.18 \\
\hline 27 & 0.0398 & 510.8 & 0.0070 & 2.22 & 54 & 0.0447 & 544.7 & 0.0185 & 4.22 \\
\hline
\end{tabular}
obtained by measured values during propagation of the Lüders bands

Table 9 Calculated values of multiregression coefficient $(R)$ and coefficient of determination $\left(R^{2}\right)$ of mathematical models of influential parameters during Lüders bands formation and propagation

\begin{tabular}{lcccc}
\hline & & \multicolumn{3}{c}{ Influential parameters } \\
\cline { 3 - 5 } & & $\sigma(\mathrm{MPa})$ & $\begin{array}{c}\varepsilon_{\max } \\
(\mathrm{mm} / \mathrm{mm})\end{array}$ & $\begin{array}{c}\Delta T_{\max } \\
\left({ }^{\circ} \mathrm{C}\right)\end{array}$ \\
\hline \multirow{2}{*}{ Formation of the Lüders band } & $R$ & 0.881 & 0.977 & \\
& $R^{2}$ & 0.776 & 0.954 & \\
\hline \multirow{2}{*}{ Propagation of the Lüders band } & $R$ & 0.871 & 0.948 & 0.968 \\
& $R^{2}$ & 0.759 & 0.899 & 0.938 \\
\hline
\end{tabular}


Table 10 Calculated results of influence parameter $\sigma$ during formation and propagation of the Lüders bands at $v=0.007 \mathrm{~s}^{-1}$

\begin{tabular}{ccc}
\hline Number of & $\begin{array}{c}\text { Formation of } \\
\text { the Lüders } \\
\text { band }\end{array}$ & $\begin{array}{c}\text { Propagation } \\
\text { of the Lüders } \\
\text { band }\end{array}$ \\
\cline { 2 - 3 } & $\begin{array}{c}\text { Output } \\
\text { parameter }\end{array}$ & $\begin{array}{c}\text { Output } \\
\text { parameter }\end{array}$ \\
\cline { 2 - 3 } & $\begin{array}{c}\sigma \\
\text { mPa) }\end{array}$ & $\begin{array}{c}\sigma \\
(\mathrm{MPa})\end{array}$ \\
\hline 1 & 532.0 & 525.6 \\
\hline 2 & 539.7 & 520.9 \\
3 & 535.9 & 533.8 \\
\hline 4 & 530.4 & 516.6 \\
5 & 514.6 & 537.3 \\
\hline 6 & 498.8 & 525.0 \\
\hline 7 & 496.6 & 486.1 \\
\hline 8 & 501.5 & 494.5 \\
\hline 9 & 537.5 & 515.5 \\
10 & 541.5 & 525.6 \\
11 & 524.5 & 524.4 \\
12 & 523.4 & 514.4 \\
\hline 13 & & 529.4 \\
\hline 14 & & 532.7 \\
\hline 15 & & 537.3 \\
\hline 16 & & 540.7 \\
\hline 17 & & 542.2 \\
\hline 18 & & 553.1 \\
\hline Deviation & $1.34 \%$ & $1.65 \%$ \\
\hline
\end{tabular}

Experimental values of the influence parameters during formation and propagation of the Lüders bands are added into the obtained mathematical models. The following calculated values of the influence parameters from the predicted models are shown in Tables 10-12.

Mathematical models of the influence parameters show low deviations of calculated values compared to the experimental results. This confirms the applicability of the obtained mathematical models in monitoring the observed influence parameters during the formation and propagation of the Lüders bands during cold deformation in niobium microalloyed steel. The functionality and reliability of mathematical models of influential parameters during formation and propagation of the Lüders bands were verified by selecting random values of the influencing parameters in the area of formation and propagation of the Lüders bands. 
Table 11 Calculated results of influence parameter $\varepsilon_{\max }$ during formation and propagation of the Lüders bands

\begin{tabular}{|c|c|c|c|c|c|c|c|}
\hline \multicolumn{4}{|c|}{ Formation of the Lüders band } & \multicolumn{4}{|c|}{ Propagation of the Lüders band } \\
\hline \multirow{2}{*}{ 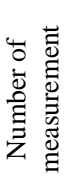 } & $\begin{array}{c}\text { Output } \\
\text { parameter }\end{array}$ & \multirow{2}{*}{ 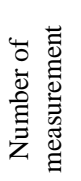 } & $\begin{array}{c}\text { Output } \\
\text { parameter }\end{array}$ & \multirow{2}{*}{ 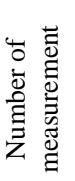 } & $\begin{array}{c}\text { Output } \\
\text { parameter }\end{array}$ & \multirow{2}{*}{ 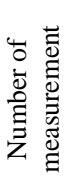 } & \multirow{2}{*}{$\begin{array}{c}\begin{array}{c}\text { Output } \\
\text { parameter }\end{array} \\
\varepsilon_{\max } \\
(\mathrm{mm} / \mathrm{mm})\end{array}$} \\
\hline & $\begin{array}{c}\varepsilon_{\max } \\
(\mathrm{mm} / \mathrm{mm})\end{array}$ & & $\begin{array}{c}\varepsilon_{\max } \\
(\mathrm{mm} / \mathrm{mm})\end{array}$ & & $\begin{array}{c}\varepsilon_{\max } \\
(\mathrm{mm} / \mathrm{mm})\end{array}$ & & \\
\hline 1 & 0.0060 & 19 & 0.0276 & 1 & 0.0369 & 28 & 0.0373 \\
\hline 2 & 0.0133 & 20 & 0.0328 & 2 & 0.0390 & 29 & 0.0373 \\
\hline 3 & 0.0242 & 21 & 0.0037 & 3 & 0.0416 & 30 & 0.0363 \\
\hline 4 & 0.0266 & 22 & 0.0106 & 4 & 0.0426 & 31 & 0.0345 \\
\hline 5 & 0.0283 & 23 & 0.0231 & 5 & 0.0408 & 32 & 0.0388 \\
\hline 6 & 0.0050 & 24 & 0.0292 & 6 & 0.0443 & 33 & 0.0415 \\
\hline 7 & 0.0096 & 25 & 0.0055 & 7 & 0.0298 & 34 & 0.0430 \\
\hline 8 & 0.0179 & 26 & 0.0207 & 8 & 0.0297 & 35 & 0.0457 \\
\hline 9 & 0.0242 & 27 & 0.0334 & 9 & 0.0309 & 36 & 0.0490 \\
\hline 10 & 0.0079 & 28 & 0.0364 & 10 & 0.0315 & 37 & 0.0357 \\
\hline 11 & 0.0257 & 29 & 0.0408 & 11 & 0.0333 & 38 & 0.0391 \\
\hline 12 & 0.0303 & 30 & 0.0103 & 12 & 0.0369 & 39 & 0.0401 \\
\hline 13 & 0.0053 & 31 & 0.0291 & 13 & 0.0316 & 40 & 0.0438 \\
\hline 14 & 0.0153 & 32 & 0.0354 & 14 & 0.0339 & 41 & 0.0441 \\
\hline 15 & 0.0251 & 33 & 0.0080 & 15 & 0.0365 & 42 & 0.0473 \\
\hline 16 & 0.0324 & 34 & 0.0230 & 16 & 0.0392 & 43 & 0.0374 \\
\hline 17 & 0.0122 & 35 & 0.0311 & 17 & 0.0396 & 44 & 0.0418 \\
\hline \multirow[t]{11}{*}{18} & 0.0188 & 36 & 0.0403 & 18 & 0.0427 & 45 & 0.0441 \\
\hline & & & & 19 & 0.0340 & 46 & 0.0454 \\
\hline & & & & 20 & 0.0388 & 47 & 0.0453 \\
\hline & & & & 21 & 0.0405 & 48 & 0.0447 \\
\hline & & & & 22 & 0.0441 & 49 & 0.0388 \\
\hline & & & & 23 & 0.0457 & 50 & 0.0410 \\
\hline & & & & 24 & 0.0500 & 51 & 0.0421 \\
\hline & & & & 25 & 0.0341 & 52 & 0.0436 \\
\hline & & & & 26 & 0.0376 & 53 & 0.0471 \\
\hline & & & & 27 & 0.0390 & 54 & 0.0485 \\
\hline & Deviation & & $12.37 \%$ & & Deviation & & $3.37 \%$ \\
\hline
\end{tabular}

Therefore, a confirmation test was performed to compare randomly selected experimental values of the influencing parameters with the calculated results obtained by predicted mathematical models, Tables 13-15.

Conducted confirmation tests have established high accuracy of the mathematical models in obtaining calculated values of the influence parameters from randomly selected experimental values during formation and propagation of the Lüders bands. 
Table 12 Calculated results of influence parameter $\Delta T_{\max }$ during propagation of the Lüders bands

\begin{tabular}{|c|c|c|c|}
\hline \multicolumn{4}{|c|}{ Propagation of the Lüders band } \\
\hline \multirow{2}{*}{ 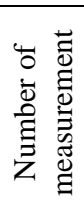 } & $\begin{array}{c}\text { Output } \\
\text { parameter }\end{array}$ & \multirow{2}{*}{ 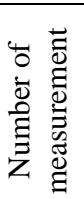 } & \multirow{2}{*}{$\begin{array}{c}\begin{array}{c}\text { Output } \\
\text { parameter }\end{array} \\
\begin{array}{c}\Delta T_{\max } \\
\left({ }^{\circ} \mathrm{C}\right)\end{array}\end{array}$} \\
\hline & $\begin{array}{c}\Delta T_{\max } \\
\left({ }^{\circ} \mathrm{C}\right)\end{array}$ & & \\
\hline 1 & 0.90 & 28 & 2.41 \\
\hline 2 & 1.09 & 29 & 2.54 \\
\hline 3 & 1.25 & 30 & 2.75 \\
\hline 4 & 1.46 & 31 & 1.81 \\
\hline 5 & 1.89 & 32 & 2.41 \\
\hline 6 & 1.78 & 33 & 2.63 \\
\hline 7 & 0.42 & 34 & 2.75 \\
\hline 8 & 0.69 & 35 & 2.58 \\
\hline 9 & 0.89 & 36 & 2.96 \\
\hline 10 & 0.99 & 37 & 2.03 \\
\hline 11 & 1.00 & 38 & 2.68 \\
\hline 12 & 1.37 & 39 & 3.09 \\
\hline 13 & 0.78 & 40 & 3.36 \\
\hline 14 & 1.09 & 41 & 3.59 \\
\hline 15 & 1.31 & 42 & 4.05 \\
\hline 16 & 1.50 & 43 & 3.05 \\
\hline 17 & 1.58 & 44 & 3.36 \\
\hline 18 & 1.81 & 45 & 3.46 \\
\hline 19 & 1.74 & 46 & 3.59 \\
\hline 20 & 2.49 & 47 & 3.79 \\
\hline 21 & 3.18 & 48 & 4.08 \\
\hline 22 & 3.33 & 49 & 2.90 \\
\hline 23 & 3.93 & 50 & 3.14 \\
\hline 24 & 4.01 & 51 & 3.48 \\
\hline 25 & 1.38 & 52 & 3.82 \\
\hline 26 & 1.71 & 53 & 4.18 \\
\hline 27 & 1.88 & 54 & 4.13 \\
\hline & Deviation & & $11.90 \%$ \\
\hline
\end{tabular}

Based on the previously obtained results and validation of the mathematical models for the influential parameters, it can be concluded that the mathematical models showed high reliability since high coefficients of multiregression and determination coefficients and low deviations of calculated results were obtained. Mathematical models are evidence that output parameter stress $(\sigma)$ can be described by maximum temperature change $\left(\Delta T_{\max }\right)$ and maximum strain $\left(\varepsilon_{\max }\right)$ considering good agreement between the experimental results and the calculated ones from polynomial models during the formation and propagation of the Lüders bands. Therefore, maximum temperature change $\left(\Delta T_{\max }\right)$ can be considered as a stress change that occurs during the formation and propagation of the Lüders bands. 
Table 13 Validation of mathematical models by the confirmation test for $\sigma$ during formation and propagation of the Lüders bands at $v=0.007 \mathrm{~s}^{-1}$

\begin{tabular}{|c|c|c|c|c|}
\hline \multirow{3}{*}{ 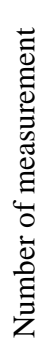 } & \multicolumn{2}{|c|}{$\begin{array}{c}\text { Formation } \\
\text { of the Lüders band }\end{array}$} & \multicolumn{2}{|c|}{$\begin{array}{c}\text { Propagation } \\
\text { of the Lüders band }\end{array}$} \\
\hline & 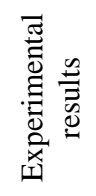 & 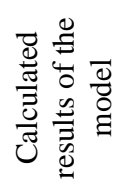 & 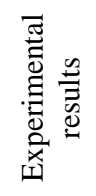 & 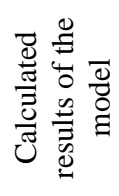 \\
\hline & $\begin{array}{c}\sigma \\
(\mathrm{MPa})\end{array}$ & $\begin{array}{c}\sigma \\
(\mathrm{MPa})\end{array}$ & $\begin{array}{c}\sigma \\
(\mathrm{MPa})\end{array}$ & $\begin{array}{c}\sigma \\
(\mathrm{MPa})\end{array}$ \\
\hline 1 & 557 & 558.7 & 525.2 & 523.8 \\
\hline 2 & 533.4 & 515.5 & 514.2 & 522.4 \\
\hline 3 & 534.6 & 560.4 & 557.3 & 547.0 \\
\hline 4 & 535.6 & 524.5 & & \\
\hline \multicolumn{2}{|c|}{ Deviation } & $2.64 \%$ & & $1.24 \%$ \\
\hline
\end{tabular}

Table 14 Validation of mathematical models by the confirmation test for $\varepsilon_{m a x}$ during formation and propagation of the Lüders bands

\begin{tabular}{|c|c|c|c|c|}
\hline \multirow{3}{*}{ 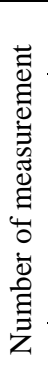 } & \multicolumn{2}{|c|}{$\begin{array}{c}\text { Formation } \\
\text { of the Lüders band }\end{array}$} & \multicolumn{2}{|c|}{$\begin{array}{c}\text { Propagation } \\
\text { of the Lüders band }\end{array}$} \\
\hline & 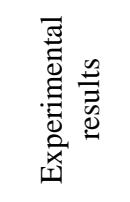 & 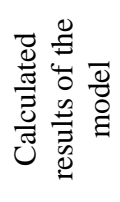 & 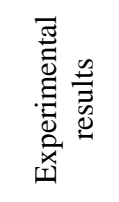 & 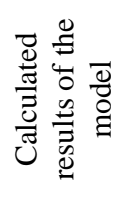 \\
\hline & $\begin{array}{c}\varepsilon \max \\
\mathrm{mm} / \mathrm{mm}\end{array}$ & $\begin{array}{c}\varepsilon \max \\
\mathrm{mm} / \mathrm{mm}\end{array}$ & $\begin{array}{c}\varepsilon \max \\
\mathrm{mm} / \mathrm{mm}\end{array}$ & $\begin{array}{c}\varepsilon \max \\
\mathrm{mm} / \mathrm{mm}\end{array}$ \\
\hline 1 & 0.0166 & 0.0215 & 0.0455 & 0.0417 \\
\hline 2 & 0.0199 & 0.0232 & 0.0355 & 0.0341 \\
\hline 3 & 0.0415 & 0.0441 & 0.0393 & 0.0403 \\
\hline 4 & 0.0392 & 0.0362 & 0.0491 & 0.0485 \\
\hline 5 & & & 0.0404 & 0.0363 \\
\hline 6 & & & 0.0457 & 0.0478 \\
\hline 7 & & & 0.0467 & 0.0466 \\
\hline 8 & & & 0.0450 & 0.0450 \\
\hline 9 & & & 0.0480 & 0.0485 \\
\hline & eviation & $15.04 \%$ & & $3.59 \%$ \\
\hline
\end{tabular}

Mathematical models have shown that the values of other influencing output parameters $\left(\varepsilon_{\max }, \Delta T_{\max }\right)$ during the formation and propagation of the Lüders bands can be described with high accuracy. It means that the behavior of niobium microalloyed steel can be predicted with models of influencing parameters in the region behind the Lüders front where maximum local temperature changes, i.e. stress changes, and strain changes are present during the cold deformation. 
Table 15 Validation of mathematical models by the confirmation test for $\Delta T_{\max }$ during propagation of the Lüders bands

\begin{tabular}{|c|c|c|}
\hline \multirow{3}{*}{ 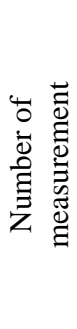 } & \multicolumn{2}{|c|}{$\begin{array}{l}\text { Propagation of the Lüders } \\
\text { band }\end{array}$} \\
\hline & 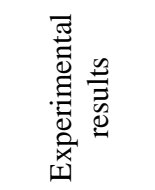 & 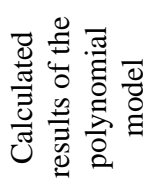 \\
\hline & $\Delta T_{\max }\left({ }^{\circ} \mathrm{C}\right)$ & $\Delta T_{\max }\left({ }^{\circ} \mathrm{C}\right)$ \\
\hline 1 & 2.02 & 2.07 \\
\hline 2 & 1.15 & 1.22 \\
\hline 3 & 2.07 & 1.71 \\
\hline 4 & 3.91 & 4.06 \\
\hline 5 & 2.22 & 2.72 \\
\hline 6 & 3.07 & 2.66 \\
\hline 7 & 4.05 & 3.92 \\
\hline 8 & 4.12 & 3.83 \\
\hline 9 & 4.01 & 4.15 \\
\hline \multicolumn{2}{|c|}{ Deviation } & $8.82 \%$ \\
\hline
\end{tabular}

Lüders bands, i.e. surface roughness, can often occur due to the effort of continuously improving of the mechanical properties (increasing yield strength and ductility) of microalloyed steels. Therefore, in order to modify and improve the metal forming process, it is important to determine the stress amounts during the Lüders band propagation.

Stress changes at different deformation degrees are visible during static tensile test in the area behind the Lüders band front by applying a mathematical model according to Eq. (6) during the propagation of the Lüders band. From the obtained results of the mathematical model, it can be concluded that the stress amount in the area behind the Lüders band front increases with increasing of deformation degree from point 1 to point 2 according to:

$$
\begin{gathered}
\sigma_{\text {point } 1, \text { Lüders band propagation }}=640.894-90.392 \cdot 0.76-1049.07 \cdot 0.0234-86.8013 \cdot 0.76^{2}- \\
399310 \cdot 0.0234^{2}+13425.7 \cdot 0.76 \cdot 0.0234=517.63 \mathrm{MPa} \\
\sigma_{\text {point } 2, \text { Lüdersbandpropagation }}=640.894-90.392 \cdot 3.42-1049.07 \cdot 0.047-86.8013 \cdot 3.42^{2}- \\
399310 \cdot 0.047^{2}+13425.7 \cdot 3.42 \cdot 0.047=543.16 \mathrm{MPa}
\end{gathered}
$$

Comparing the stress amounts at the start of the Lüders band propagation (point 1) and during the Lüders band propagation (point 2), a higher stress amount of 543.16 MPa was determined at point 2 at a higher deformation degree. A higher stress amount indicates a more pronounced surface roughness in this area during Lüders band propagation which is a significant problem during forming of microalloyed steels.

The possibility of the stress amounts prediction with the obtained mathematical models is proven. For example, during cold drawing and bending of microalloyed pipes with inhomogeneous deformations, the significance of the surface roughness can be predicted and so can the possibilities of plastic flow of niobium microalloyed steel from obtained 
stress amounts. Predicting of the stress amounts in steels, with the Lüders band appearance, can also be significant during sheet forming, especially sheet metal drawing, and deep drawing in automobile production.

It has been proved that the obtained mathematical models can improve the product quality and reduce the costs of the forming process of microalloyed steels since the calculation of the values of the influential parameters by using predicted models do not necessarily require additional experimental tests requiring additional costs.

\section{CONCLUSION}

Mathematical models of the influence parameters during the formation and propagation of the Lüders bands in the area behind the Lüders band front in niobium microalloyed steel are developed in this paper. The proposed mathematical models give a new approach to the prediction of stresses, maximum temperature changes and strains during the Lüders band formation and propagation in niobium microalloyed steel.

High reliability of the obtained mathematical models are determined with high coefficients of multiregression within the range from 0.871 to 0.977 , low deviations of calculated results from $1.34 \%$ to $12.37 \%$ and low deviations of confirmation results from $1.24 \%$ to $15.04 \%$.

It has been proved that output parameter stress $(\sigma)$ can be described by maximum temperature change $\left(\Delta T_{\max }\right)$ and maximum strain $\left(\varepsilon_{\max }\right)$ where maximum temperature change $\left(\Delta T_{\max }\right)$ can be considered as a stress change during the formation and propagation of Lüders bands.

Stress amounts at different deformation degrees, in the deformation zone during Lüders band propagation, were determined for modification and improvement of steel forming processes. Using the obtained mathematical model, it was observed that the stress changes at different deformation degrees during static tensile tests in the area behind the Lüders band front. At the start of the Lüders band propagation, a lower stress amount of 517.63 $\mathrm{MPa}$, is determined with respect to the advanced Lüders band propagation stress amount of 543.16 MPa. A higher stress amount, obtained by the proposed mathematical model, indicates a more pronounced surface roughness in the area of the Lüders band propagation.

The proposed mathematical models can find their application in the industry for improving the forming process by prediction of the surface roughness and plastic flow of the tested steel using the obtained amounts of influence parameters, especially stress amounts. The significance of the surface roughness as well as the possibilities of plastic flow of niobium microalloyed steel can be predicted by the proposed mathematical models during sheet forming and deep drawing in automobile production as well as in cold drawing and bending of microalloyed pipes for pipelines with inhomogeneous deformations.

Application of the obtained mathematical models of influence parameters can reduce costs in the forming process while no additional experimental tests are required.

Acknowledgements: This work is financially supported by the Croatian Science Foundation under project number IP-2016-06-1270 (Principal Investigator: Prof.dr.sc. Stoja Rešković) and by the University of Rijeka, Croatia, contract number uniri-tehnic-18-100-1235. 


\section{REFERENCES}

1. Kumar, R., Rajesh Jesudoss Hynes, N., 2020, Prediction and optimization of surface roughness in thermal drilling using integrated ANFIS and GA approach, Engineering Science and Technology, An International Journal, 23, pp. 30-41.

2. Zębala, W., 2010, Milling optimization of difficult to machine alloys, Management and Production Engineering Review, 1(1), pp. 59-70.

3. Zhou, T., Zurob, H., Zhang, P., Kuuskman, K., Cho, S.H., Burella, D., 2019, Control of edge breaks during cold mill processing of commercial and drawing quality low-carbon steels, Ironmaking \& Steelmaking, 46, pp. 656-662.

4. Barišić, B., Pepelnjak, P., Math, M.D., 2008, Predicting of the Lüders' bands in the processing of TH material in computer environment by means of stochastic modeling, Journal of Materials Processing Tehnology, 203, pp. 154-165.

5. Torkamani, H., Raygan, S., Garcia Mateo, C., Rassizadehghani, J., Palizdar, Y., San-Martin, D., 2019, Tensile behavior of normalized low carbon $\mathrm{Nb}$-microalloyed steel in the presence of rare earth elements, Materials Science and Engineering: A, 749, pp. 56-64.

6. Petit, J., Wagner, D., Ranc, N., Montay, G., François, M., 2013, Comparison of different techniques for the monitoring of the Lüders bands development, $13^{\text {th }}$ International Conference on Fracture ICF13, Beijing, China, pp. 3797-3809.

7. Brlić, T., Rešković, S., Jandrlić, I., 2020, Influence of niobium content on strain amount in Lüders bands in niobium microalloyed steel, Metals and Materials International, 26(2), pp. 179-187.

8. Rešković, S., Brlić, T., Jandrlić, I., Vodopivec, F., 2020, Influence of strip cooling rate on Lüders bands appearance during subsequent cold deformation, New Technologies, Development and Application II, Springer Nature, Cham, Switzerland, pp. 115-121.

9. Pepelnjak, T., Barisic, B., 2007, Analysis and elimination of the stretcher strains on TH415 tinplate rings in the stamping process, Journal of Materials Processing Technology, 186(1-3), pp. 111-119.

10. Bhirud, N.L., Gawande, R.R., 2017, Optimization of process parameters during end milling and prediction of work piece temperature rise, Archive of Mechanical Engineering, 64(3), pp. 327-346.

11. Haneef, T., Lahiri, B.B., Bagavathiappan, S., Mukhopadhyay, C.K., Philip, J., Chandra Rao, B.P., Jayakumar T., 2015, Study of the tensile behavior of AISI type 316 stainless steel using acoustic emission and infrared thermography techniques, Journal of Materials Research and Technology, 4(3), pp. 241-253.

12. Nasri, M.T., Slimani, F., Ayadi, M., Cherouat, A., Rezgui, M., Zghal, A., 2012, Performance evaluation of multiple regression method for identification models: Application to the sheet metal forming formability, Advanced Materials Research, 423, pp. 177-186.

13. Jurković, M., Jurković, Z., Buljan, S., Obad, M., 2018, An experimental and modelling approach for improving utilization rate of the cold roll forming production line, Advances in Production Engineering \& Management, 13, pp. 57-68.

14. Aherwar, A., Unune, D., Pathri, B., Kishan, J., 2014, Statistical and regression analysis of vibration of carbon steel cutting tool for turning of EN24 steel using design of experiments, International Journal of Recent advances in Mechanical Engineering, 3(3), pp. 137-151.

15. Jurković, Z., Cukor, G., Brezocnik, M., Brajkovic, T., 2018, A comparison of machine learning methods for cutting parameters prediction in high speed turning process, Journal of Intelligent Manufacturing, 29, pp. 1683-1693.

16. Li, L., Li, Y., Yang, M., Tong, T., 2019, Process parameters decision to optimization of cold rolling-beating forming process through experiment and modelling, Metals, 9(4), pp. 405.

17. Achebo, J., 2015, Development of a predictive model for determining mechanical properties of AA 6061 using regression analysis, Production \& Manufacturing Research: An Open Access Journal, 3(1), pp. 169-184.

18. Balokhonov, R.R., Romanova, V.A., 2007, Numerical modeling of the thermomechanical behavior of steels with allowance for the propagation of Luders bands, Journal of Applied Mechanics and Technical Physics, 48(5), pp. 743-750.

19. Barišić, B., 2005, Analiza pojave Lüdersovih traka u procesu izrade proizvoda iz tankostjenog lima, Doctoral Thesis at the University of Rijeka, Faculty of Engineering, Rijeka, Croatia

20. Sadowski, A.J., Rotter, J.M., Stafford, P.J., Reinke, T., Ummenhofer, T., 2017, On the gradient of the yield plateau in structural carbon steels, Journal of Constructional Steel Research, 130, pp. 120-130.

21. Rešković, S., Grizelj, B., Brlić, T., Balenović, M., 2019, The application of a factorial experiment with repeated measurements in the investigation of parameters influencing the properties of niobium microalloyed cold drawn welded steel tubes, Transactions of FAMENA, 43(4), pp. 17-26. 